\title{
Nonspecific chest pain and hospital revisits within 7 days of care: variation across emergency department, observation and inpatient visits
}

\author{
Grant R. Martsolf ${ }^{1,2}$, Teryl K. Nuckols ${ }^{3,4}$, Kathryn R. Fingar ${ }^{5}$, Marguerite L. Barrett ${ }^{6}$, Carol Stocks ${ }^{7,8}$ and \\ Pamela L. Owens ${ }^{9^{*}}$
}

Keywords: Chest pain, Hospital, Readmission, Observation services, Acute care, Emergency department, Insurance

\section{Background}

Patients increasingly receive care in outpatient observation services rather than being admitted to the hospital, and chest pain is the leading diagnosis among observed patients [1-4]. Chest pain can indicate a serious medical condition (e.g., acute coronary syndromes, including acute myocardial infarction [AMI] and unstable angina) or a relatively minor one (e.g., gastroesophageal reflux). Often the difference between a serious and minor condition is not readily apparent $[5,6]$. A relatively small percentage of patients who present to the hospital with chest pain have acute coronary syndromes or other emergent conditions requiring inpatient care [7]. However, determining the diagnosis and appropriate course of treatment for chest pain can involve clinical evaluation, testing, and monitoring. Clinicians historically had two options: hold patients with chest pain in the emergency department (ED), or admit them to the hospital for a short stay. Observation status offers a third option, allowing more time to assess the patient's condition.

The concept of hospital observation emerged in the 1970 s as an alternative to ED and inpatient care $[8,9]$. For chest pain, randomized controlled trials in the 1990s and 2000s demonstrated that dedicated cardiac

\footnotetext{
* Correspondence: pam.owens@ahrq.hhs.gov

${ }^{9}$ Agency for Healthcare Research and Quality, 5600 Fishers Lane, Rockville, MD 20857, USA

Full list of author information is available at the end of the article
}

observation units and specific care protocols improved outcomes, reduced hospital admissions, and lowered costs [10-13]. The practice of hospital observation status has evolved over time, and expanded in diverse ways across different states and payers. Many public and private payers now reimburse observation services as a hospital-based, outpatient service irrespective of diagnosis, use of a specific protocol, or physical location in the hospital [14, 15]. The Centers for Medicare \& Medicaid Services defines observation services as "a well-defined set of specific, clinically appropriate services, which include ongoing short term treatment, assessment, and reassessment before a decision can be made regarding whether patients will require further treatment as hospital inpatients or if they are able to be discharged from the hospital." [16]

Despite rapid growth of observation services for chest pain $[17,18]$, it is unclear whether observation services in current practice is associated with different outcomes compared with ED or inpatient care [19]. Unlike the historical randomized controlled trials previously mentioned, many hospitals do not have designated observation units or use chest pain protocols [18]. A potentially useful outcome measure following an ED, observation, or inpatient hospital visit is the percentage of patients who return to the hospital [20, 21]. Compared with longer follow-up periods, a 7-day follow-up period emphasizes revisits that are more likely to involve acute 
issues related to the index visit [22, 23]. Moreover, the first week after a hospital visit for chest pain represents a high-risk period during which atherosclerotic heart disease and AMI are leading causes of death [23, 24]; thus, return visits for acute coronary syndromes are particularly concerning.

Our primary objective was to compare the rate at which patients with nonspecific chest pain return to the hospital within 7 days after index observation visits versus after index ED and inpatient visits. Although acuity likely differs across these three types of visits, no benchmarks have been established for assessing outcomes of observation care, making ED and inpatient care useful points of comparison [25]. Secondary objectives included examining [1] the type of revisit (ED, observation, or inpatient) to determine how often patients receive a higher level of care upon return and [2] first-listed diagnoses at the revisit to assess whether patients may be returning for acute coronary syndromes, nonspecific and other causes of chest pain, or other reasons.

\section{Methods}

\section{Study data}

We used 2013 and 2014 data from the Healthcare Cost and Utilization Project (HCUP) [26] State Inpatient Databases (SID), State Emergency Department Databases (SEDD), and State Ambulatory Surgery and Services Databases (SASD) [27]. We selected 10 states (Georgia, Iowa, Maryland, Nebraska, Nevada, South Carolina, South Dakota, Tennessee, Vermont, and Wisconsin) because they had complete data on observation services and encrypted patient linkage numbers that linked index visits and revisits within the same state. These states represented 13\% of the U.S. population in 2014 (4\% West, 23\% South, 17\% Midwest, and 1\% Northeast).

\section{Study population}

We included patients aged 18 years or older with an index visit for a first-listed (or principal) diagnosis of nonspecific chest pain. The unit of analysis was an index visit-ED visit, short-stay observation visit, or short-stay inpatient visit (defined below). Patients with multiple encounters contributed multiple index visits if the index visit met the following exclusion and inclusion criteria. We excluded index visits if the patient transferred into or out of the hospital, died in the hospital, or left against medical advice. We required that index visits have a clean period, defined as no hospitalization, observation visit, or ED visit for any reason in the past 30 days [28]. To identify events that occurred before and after the index visit, we only included index visits that occurred between February 2013 and November 2014.

\section{Independent variable}

The primary independent variable was the type of index visit for nonspecific chest pain:

1. Index ED visit. The patient was discharged from ED care without being placed under observation or admitted.

2. Index observation visit. The patient was discharged from observation care within 2 nights after presentation to the hospital without being admitted.

3. Index inpatient visit. The patient was discharged from short-stay inpatient care within 2 nights after presentation to the hospital.

Determination of type of index visit was based on status at time of discharge (e.g., patients seen in the ED but then moved to observation were considered having an observation visit. We included only short-stay observation and inpatient visits (discharge within 2 nights [15, 29] after presentation to the hospital) to increase the comparability of the three types of visits.

\section{Dependent variables}

The primary dependent variable was the presence or absence of any revisit to the hospital-meaning ED, observation, or inpatient visits-within 7 days of the discharge date from the index visit. Secondary dependent variables included type of care (ED, observation, or inpatient) and diagnosis at the revisit. For the latter, we created a categorical variable based on the following hierarchy: (1) any diagnosis of acute coronary syndromes (AMI or unstable angina), (2) any diagnosis of nonspecific chest pain and other conditions potentially associated with chest pain (e.g., other cardiac conditions, lower respiratory disease, esophageal disorders), and (3) unrelated conditions (defined in Additional file 1 Table S1) [5].

\section{Covariates}

Covariates included patient sex, age, expected primary payer (Medicare, Medicaid, private, self-pay / no charge), urban/rural location, median household income quartile (derived using patient ZIP Code), as well as coexisting conditions at the index visit: prior AMI, any other ischemic heart disease, other comorbid conditions identified using Elixhauser Comorbidity software [30], designed to predict 30-day readmissions. We included 14 out of 29 comorbid conditions, which represented at least $1 \%$ of all index records in all three settings. We also included a variable representing the number of diagnoses reported on the record, because coexisting conditions may be documented more frequently for inpatient and observation than for ED visits. 


\section{Analysis}

We estimated separate logistic regression models for the primary and secondary dependent variables including the covariates described above and hospital-level fixed effects, because care may be influenced by unknown factors that are constant across visits to a particular hospital.

We used results from the models to derive riskadjusted 7-day revisit rates for different types of index visits, applying a widely used approach for risk adjustment (described in the Additional file) [31]. We repeated these analyses stratifying by expected primary payer, because insurance is likely to influence access to ambulatory care after a hospital visit. We also repeated these analyses using the secondary outcome of diagnosis at the revisit. Because large sample sizes increase the probability of a type I error, we considered results statistically significant if the $p$-value was $<0.01$ and the revisit rates differed by $10 \%$ or more.

\section{Results}

\section{Sample characteristics}

We included 1,005,866 index visits to 746 hospitals for nonspecific chest pain. Most index visits occurred in the ED $(638,302)$ with about half as many index observation visits $(325,770)$ and fewer index inpatient visits $(41,794)$. Compared with patients with index observation visits, patients with index inpatient visits were more likely to be older, have Medicare insurance, and have more documented coexisting conditions, whereas patients with index ED visits were more likely to be younger, have private insurance, and have fewer coexisting conditions (Table 1).

There were 86,692 revisits $(62,629$ following index ED visits, 20,472 following index observation visits, and 3591 following index inpatient visits). Total unadjusted 7 -day revisit rates after index visits for ED care, observation care, and inpatient care were 9.8, 6.3, and 8.6\%, respectively (Fig. 1). Regardless of the type of index visit, over half of 7-day revisits had been discharged from the ED without observation or inpatient care. The rates at which patients returned for inpatient care were $1.5 \%$ among patients with an index visit for ED care, $1.2 \%$ among patients with index observation visits, and $2.7 \%$ among patients with index inpatient visits.

\section{Seven-day risk-adjusted revisit rate}

Adjusted revisit rates following an index visit for nonspecific chest pain involving ED care, observation care, and inpatient care were 9.7\% (95\% CI: 9.7-9.8), 6.5\% (95\% CI: 6.4-6.6), and 7.7\% (95\% CI: 7.5-8.0), respectively (Fig. 2). The risk-adjusted 7-day revisit rate was 3.2 (95\% CI: 3.1-3.3) and 1.2 (95\% CI, 1.0-1.5) percentage points lower, respectively, following an index observation visit than following an index $\operatorname{ED}(p<0.001)$ or inpatient $(p<0.001)$ visit.

\section{Revisit rates by payer population}

Results were similar across payers (Fig. 2). Relative to the risk-adjusted 7-day revisit rate following an index ED visit, the rate following an index observation visit was 5.1 (95\% CI: 4.9-5.3) percentage points lower among patients with Medicare $(p<0.001), 3.7$ (95\% CI: 3.3-3.9) percentage points lower among patients with Medicaid ( $p<0.001), 1.9$ (95\% CI: 1.7-2.1) percentage points lower among patients with private insurance $(p<$ 0.001 ), and 2.6 (95\% CI: $2.2-2.8$ ) percentage points lower among patients with no insurance $(p<0.001)$. Relative to the risk-adjusted 7-day revisit rate following an index inpatient visit, the rate following an index observation visit was 1.1 (95\% CI: 0.7-1.5) percentage points lower among patients with Medicare $(p<0.001), 0.8$ (95\% CI: 0.3-1.4) percentage points lower among patients with private insurance $(p<0.01)$, and 1.7 (95\% CI: $0.9-2.5)$ percentage points lower among patients with no insurance $(p<0.001)$. Among patients with Medicaid, revisit rates were similar after index visits for observation and inpatient care.

\section{Diagnosis at revisit}

Revisits for acute coronary syndromes were rare (0.3 to $0.5 \%$ of index visits), while revisits for nonspecific chest pain and conditions potentially associated with chest pain were common (4.7 to $7.2 \%$ of index visits), irrespective of the type of index visit (Fig. 3). The riskadjusted rate at which patients returned within 7 days with acute coronary syndromes was 0.2 (95\% CI: $0.1-$ 0.2 ) and 0.1 (95\% CI: $0.0-0.2)$ percentage points lower, respectively, after an index observation visit than after an index ED visit $(p<0.001)$ and after an index inpatient visit $(p<0.001)$. Additionally, the risk-adjusted rate at which patients returned within 7 days with nonspecific chest pain or conditions potentially associated with chest pain were 2.5 (95\% CI: $2.4-2.6)$ and 0.9 (95\% CI: $0.6-$ 1.1) percentage points lower, respectively, after an index observation visit than after an index $\operatorname{ED~}(p<0.001)$ or inpatient $(p<0.001)$ visit.

Patients most frequently returned to the hospital with nonspecific chest pain or conditions potentially associated with chest pain. The risk-adjusted 7-day revisit rate following an index ED visit was 9.7\% (95\% CI: 9.7-9.8), including a $7.2 \%$ (95\% CI: 7.2-7.3) revisit rate for nonspecific chest pain or conditions potentially associated with chest pain (i.e., $74.3 \%$ of total revisits). The results from Fig. 3 were largely consistent across payers (not shown). 
Table 1 Nonspecific Chest Pain Index Visit Characteristics, by Type of Index Visit, 2013 and 2014

\begin{tabular}{llll}
\hline Characteristic & \multicolumn{3}{l}{ Type of Index Visit } \\
\cline { 2 - 4 } & ED & Observation & Inpatient \\
\hline Total, N & 638,302 & 325,770 & 41,794 \\
Originated in the ED & 638,302 & 313,000 & 37,363 \\
Direct admission & N/A & 12,770 & 4431 \\
Female, \% & 57.8 & 57.0 & 52.8 \\
Age, years, mean & 46.6 & 57.9 & 60.0 \\
Expected primary payer, \% & & & \\
$\quad$ Medicare & 23.7 & 38.7 & 47.3 \\
$\quad$ Medicaid & 17.1 & 10.7 & 10.3 \\
$\quad$ Private & 36.3 & 35.3 & 27.5 \\
$\quad$ Self-pay / No charge & 18.8 & 11.5 & 10.5 \\
$\quad$ Other & 3.9 & 3.5 & 4.2
\end{tabular}

Patient's income quartile, \%

$\begin{array}{llll}\text { Quartile 1 (poorest) } & 35.5 & 34.6 & 35.9 \\ \text { Quartiles 2 and 3 } & 49.5 & 47.7 & 48.8 \\ \text { Quartile 4 (wealthiest) } & 13.6 & 16.2 & 13.7 \\ \text { ocation of residence, \% } & & & \\ \text { Large metropolitan } & 37.7 & 42.7 & 42.4 \\ \text { Small metropolitan } & 39.6 & 34.6 & 36.2 \\ \text { Micropolitan } & 12.7 & 12.6 & 12.7 \\ \text { Rural } & 9.8 & 9.9 & 8.5 \\ \text { Diagnoses reported on } & 3.9 & 8.3 & 10.4\end{array}$

the index visit, mean $\mathrm{N}$

Most frequent coexisting condition, $\%^{\mathrm{a}}$

Elixhauser Comorbidities ${ }^{b}$

\begin{tabular}{llll} 
Alcohol abuse & 1.4 & 2.7 & 4.7 \\
$\begin{array}{l}\text { Chronic pulmonary } \\
\text { disease }\end{array}$ & 8.8 & 16.8 & 21.6 \\
$\begin{array}{l}\text { Congestive heart } \\
\text { failure }\end{array}$ & 2.5 & 5.9 & 12.0 \\
$\begin{array}{l}\text { Deficiency anemias } \\
\text { Depression }\end{array}$ & 1.4 & 5.6 & 11.0 \\
$\begin{array}{l}\text { Diabetes } \\
\text { Drug abuse }\end{array}$ & 3.2 & 10.7 & 13.0 \\
$\begin{array}{l}\text { Fluid/electrolyte } \\
\text { disorders }\end{array}$ & 10.7 & 25.5 & 33.1 \\
$\begin{array}{l}\text { Hypertension } \\
\text { Hypothyroidism }\end{array}$ & 2.3 & 2.9 & 4.4 \\
Obesity & 2.4 & 9.6 & 15.5 \\
$\begin{array}{l}\text { Other neurological } \\
\text { disorders }\end{array}$ & 1.7 & 65.6 & 75.6 \\
$\begin{array}{l}\text { Psychoses } \\
\text { Renal failure }\end{array}$ & 1.9 & 14.6 & 12.2 \\
Red to heart disease & 1.5 & 5.4 & 19.9 \\
\hline
\end{tabular}

Related to heart disease
Table 1 Nonspecific Chest Pain Index Visit Characteristics, by Type of Index Visit, 2013 and 2014 (Continued)

\begin{tabular}{clll}
\hline Characteristic & \multicolumn{3}{l}{ Type of Index Visit } \\
\cline { 2 - 4 } & ED & Observation & Inpatient \\
\hline $\begin{array}{c}\text { Other ischemic } \\
\text { heart disease }\end{array}$ & 6.3 & 24.4 & 41.2 \\
Prior AMI & 3.4 & 8.5 & 13.7 \\
Revisits, N & 62,629 & 20,472 & 3591 \\
\hline
\end{tabular}

Abbreviations: AMI Acute myocardial infarction, ED Emergency department, $N$ Number, N/A Not applicable

Note: Percentages may not sum to $100 \%$ because some index visits are missing the information

${ }^{a}$ Coexisting conditions include those from the Elixhauser comorbidity software and those related to heart disease. Only conditions with at least $1 \%$ of records in all three settings are shown. The diabetes measure includes diabetes with and without complications

${ }^{\mathrm{b}}$ The following indicators were defined using the Elixhauser Comorbidity Software, the specifications of which can be found

at https://www.hcup-us.ahrq.gov/toolssoftware/comorbidity/comorbidity.jsp 'Defined using the ICD-9 CM code 414, Other forms of chronic ischemic heart disease

Source: Agency for Healthcare Research and Quality, Healthcare Cost and Utilization Project, State Inpatient Databases, State Emergency Department Databases, and State Ambulatory Surgery and Services Databases, 10 states (Georgia, lowa, Maryland, Nebraska, Nevada, South Carolina, South Dakota,

Tennessee, Vermont, Wisconsin), 2013 and 2014

\section{Discussion}

The present analysis of hospital-based visits for nonspecific chest pain has two notable findings. First, nearly 1 in 10 patients discharged from the ED and 1 in 13 patients discharged following an inpatient hospitalization returned to the hospital within 7 days of the index visit. Second, compared with ED and inpatient visits, observation visits for nonspecific chest pain were associated with lower revisit rates ( 1 in 15).

Risk stratification for acute coronary syndromes is a critical task in the evaluation of patients with chest pain. Reassuringly, very few returning patients in our analysis $(<0.5 \%)$ had acute coronary syndromes, suggesting that prevailing hospital practices effectively identify patients at low short-term risk. These results are consistent with a recent clinical trial [32]. In that study, patients presenting to the ED with acute chest pain, non-ischemic electrocardiograms, and negative biomarkers were at low risk of major adverse cardiac events in the subsequent month whether or not they underwent non-invasive stress testing [32].

Although patients rarely returned with acute coronary syndromes, we found that many came back with nonspecific chest pain or conditions associated with chest pain. The high rate of 7-day revisits for these recurrent, uncontrolled symptoms raise concerns-for reasons similar to the problems associated with high 30-day readmission rates for a variety of conditions following inpatient hospitalization. Hospital revisits represent potentially avoidable healthcare utilization, they may contribute to ED and hospital overcrowding, and, most importantly, they demonstrate that patients have unmet needs for 


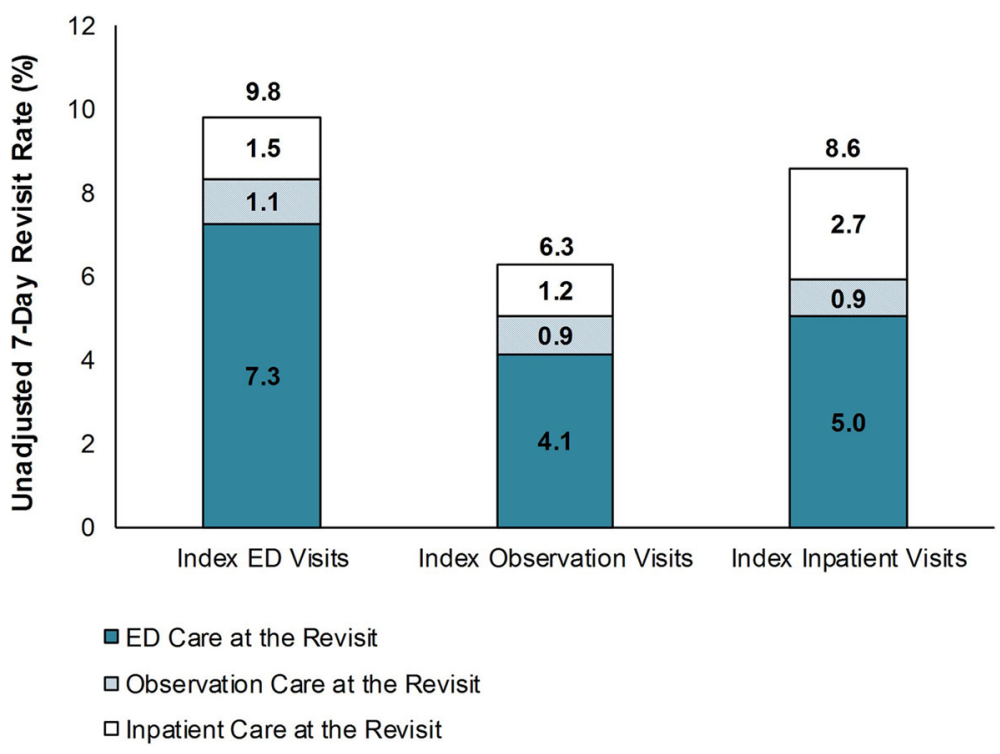

Fig. 1 Unadjusted 7-Day Revisit Rate, by Type of Index Visit, 2013 and 2014. Abbreviation: ED, emergency department. Source: Agency for Healthcare Research and Quality, Healthcare Cost and Utilization Project, State Inpatient Databases, State Emergency Department Databases, and State Ambulatory Surgery and Services Databases, 10 states (Georgia, lowa, Maryland, Nebraska, Nevada, South Carolina, South Dakota, Tennessee, Vermont, Wisconsin), 2013 and 2014

care. The frequency of revisits raises questions about healthcare delivery factors including the quality of care at the index visit, the transition from the index visit to follow-up care, and access to outpatient care after the index visit. In addition to healthcare delivery factors, diverse patient factors may be associated with 7-day revisit rates including age, comorbidity, income, health insurance, social supports, health literacy, self-efficacy in managing symptoms, language barriers, and other social determinants of health.

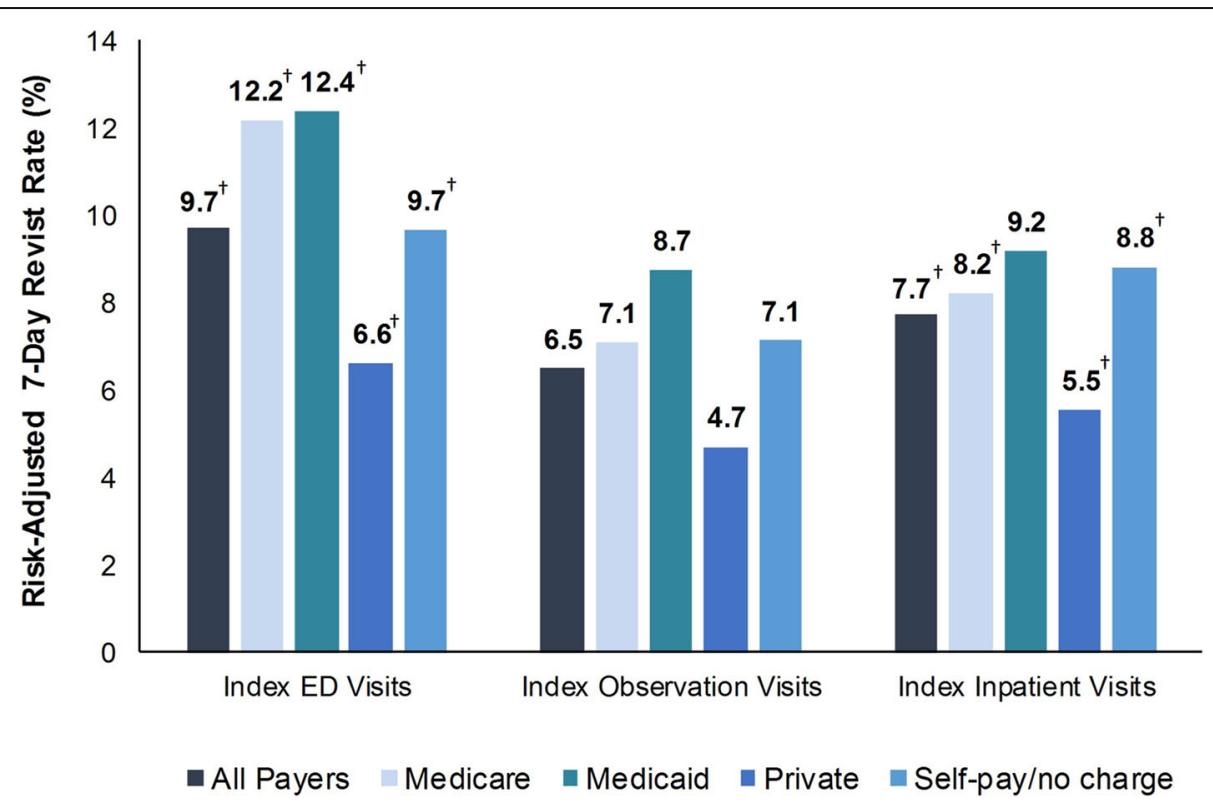

Fig. 2 Risk-Adjusted 7-Day Revisit Rate, by Payer Population and Type of Index Visit, 2013 and 2014. Abbreviation: ED, emergency department. ${ }^{\dagger}$ Statistically significant at $p<0.05$ and $\geq 10 \%$ difference between revisit rates for index observation visits and index ED or inpatient visits. Source: Agency for Healthcare Research and Quality, Healthcare Cost and Utilization Project, State Inpatient Databases, State Emergency Department Databases, and State Ambulatory Surgery and Services Databases, 10 states (Georgia, lowa, Maryland, Nebraska, Nevada, South Carolina, South Dakota, Tennessee, Vermont, Wisconsin), 2013 and 2014 


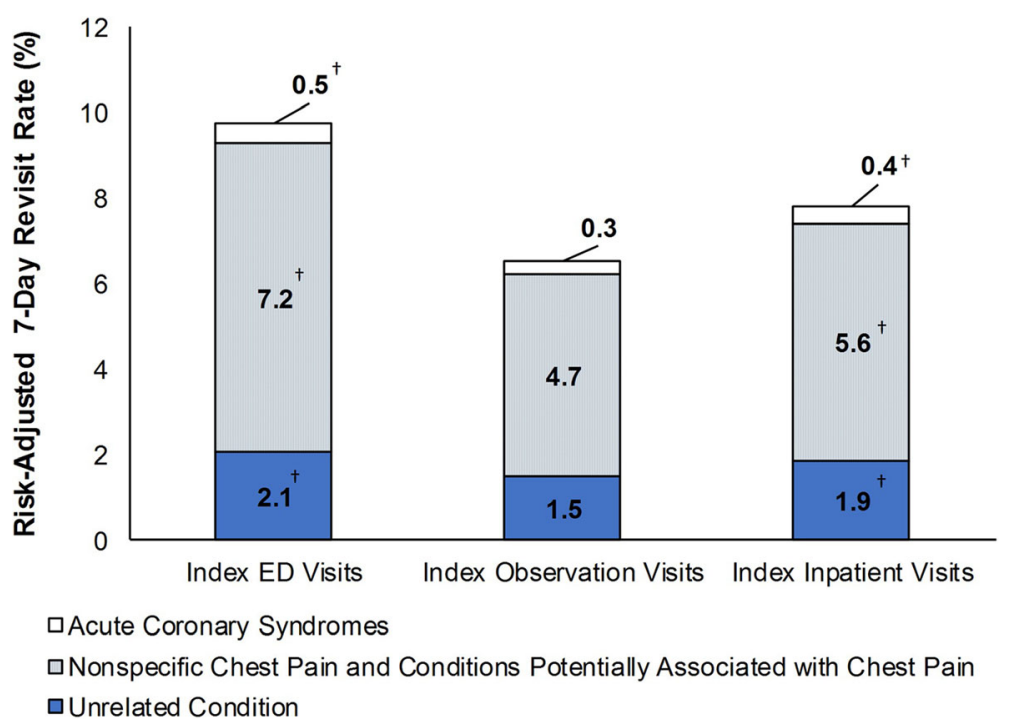

Fig. 3 Diagnosis at Revisit: Risk-Adjusted 7-Day Revisit Rates by Type of Index Visit, 2013 and 2014. Abbreviation: ED, emergency department. ${ }^{\dagger}$ Statistically significant at $p<0.05$ and $\geq 10 \%$ difference between revisit rates for index observation visits and index ED or inpatient visits. Source: Agency for Healthcare Research and Quality, Healthcare Cost and Utilization Project, State Inpatient Databases, State Emergency Department Databases, and State Ambulatory Surgery and Services Databases, 10 states (Georgia, lowa, Maryland, Nebraska, Nevada, South Carolina, South Dakota, Tennessee, Vermont, Wisconsin), 2013 and 2014

Another factor potentially contributing to revisit rates is that clinical standards of care for nonspecific chest pain remain poorly defined. Clinical practice guidelines emphasize ruling out acute coronary syndromes and other acute life-threatening conditions, but provide less information on how to evaluate and manage nonspecific and noncoronary causes of chest pain [5,33]. After acute coronary syndromes have been excluded, a comprehensive work-up often reveals that patients with nonspecific chest pain have gastroesophageal or chest wall conditions [34]. One concern of note is that the one-year mortality among patients with nonspecific chest pain is similar to that of patients with acute coronary syndromes, and $40 \%$ of patients with nonspecific chest pain have underlying coronary heart disease [35]. Thus, future research is needed to refine clinical standards of care for this population beyond the current focus on ruling out acute coronary syndromes. Furthermore, in addition to clinical decision-making, understanding the role of the healthcare delivery system and patient factors on revisit rates is important. For example, the decision to place patients with chest pain under observation can also be influenced by the availability of inpatient beds or whether family support is available if the patient were to be discharged.

Although originally designed to avoid unnecessary inpatient hospitalizations, observation care may allow clinicians a greater opportunity to diagnose noncardiac causes of chest pain and to educate patients in the selfmanagement of symptoms. We found that, relative to ED visits, 7-day revisit rates were 3.2 percentage points lower ( 33.0\%) across all populations and 5.1 percentage points lower $(-41.8 \%)$ among Medicare patients following index observation visits. Given our study design, these differences could be due to selection bias in the populations placed under observation vs. discharged from the ED. However, our findings were similar with and without adjustment, across payer populations, and by diagnosis at the return visit. Moreover, observed patients were older than those discharged from the ED, suggesting that they would be expected to have higher, not lower, revisit rates.

The results of our study are similar to two previous studies that examined outcomes after hospital visits for chest pain among Medicare beneficiaries [36] and patients within the Veteran's Affairs system [29]. Both studies found that patients cared for in observation were $7-12 \%$ less likely to return to the hospital than those who received inpatient care. The study of Medicare beneficiaries found a $29 \%$ reduction in the risk of readmission for observed patients compared with those cared for in the ED. [36] These studies focused on 30day revisits as opposed to 7-day revisits, so we cannot directly compare results. Our study extends the literature by including all adult patients and payers (including self-pay/no charge). We found similar patterns across payer populations, though the differences in revisit rates between observation and ED care were largest for Medicare patients.

Our study has several limitations. First, coding for coexisting conditions likely differs across ED, observation, and 
inpatient visits. For example, obesity was coded more often during inpatient and observation visits than during ED visits. We re-ran our models with and without conditions for which documentation varied across types of visits, as well as with and without the number of secondary conditions listed on the record, and results were similar. Second, risk-adjusted revisit rates may be higher for inpatient care than observation care because of differences in severity that are not observable in our dataset. For instance, claims data lack clinical information, such as positive cardiac biomarkers, which would reflect the risk of acute coronary syndromes. We were unable to identify revisits for patients who sought care in states not included in this study and we were unable to link records from patients who visited hospitals in multiple states. Finally, the fact that placing some patients under observation is associated with lower revisit rates does not imply that more patients with chest pain should be placed under observation in the future. Rather, the high revisit rates for nonspecific chest pain warrant thorough investigation, including rigorous testing of any future interventions designed to reduce revisits.

\section{Conclusion}

In conclusion, up to 1 in 10 patients discharged with nonspecific chest pain returned to the hospital within 1 week. Compared with ED and inpatient care, observation visits were associated with lower revisit rates. The diagnoses at revisits were largely nonspecific chest pain and conditions associated with chest pain; acute coronary syndromes were very rare. The high rates of revisits demonstrate that patients may have unmet needs for care going beyond the need to rule out acute coronary syndromes. Future research is needed to refine clinical standards of care for nonspecific chest pain as well as investigate the healthcare delivery and patient factors that influence 7-day revisit rates.

\section{Supplementary information}

Supplementary information accompanies this paper at https://doi.org/10. 1186/s12913-020-05200-x.

Additional file 1. Appendix Table S1. Risk Adjustment Method and

Conditions at the Revisit by Type of Index Visit.

\section{Abbreviations}

AHRQ: Agency for Healthcare Research and Quality; AMI: Acute myocardial infarction; CCS: Clinical Classifications Software; Cl: Confidence interval; ED: emergency department; HCUP: Healthcare Cost and Utilization Project; N: Number; N/A: Not applicable; SASD: State Ambulatory Surgery and Services Databases; SEDD: State Emergency Department Databases; SID: State Inpatient Databases

\section{Acknowledgments}

The authors gratefully acknowledge Minya Sheng, M.S. for assistance in programming and data management and Linda Lee, Ph.D. for providing editorial review of the manuscript. We also wish to acknowledge the 10
HCUP Partner organizations that contributed to the HCUP State Databases used in this study: Georgia Hospital Association, lowa Hospital Association, Maryland Health Services Cost Review Commission, Nebraska Hospital Association, Nevada Department of Health and Human Services, South Carolina Revenue and Fiscal Affairs Office, South Dakota Association of Healthcare Organizations, Tennessee Hospital Association, Vermont Association of Hospitals and Health Systems, Wisconsin Department of Health Services.

\section{Authors' contributions}

$\mathrm{GM}, \mathrm{TN}, \mathrm{KF}, \mathrm{MB}, \mathrm{CS}$ and $\mathrm{PO}$ contributed to the concept and design of the study. CS and PO contributed to acquisition of the data and funding. KF, MB, CS and PO contributed to the analysis of the data. GM, TN, KF, MB, CS and $\mathrm{PO}$ contributed to the interpretation of the data, drafting of the manuscript, critical revision, and final approval for publication. CS, PO, and KF provided administrative support and project management. All authors have read and approved the manuscript.

\section{Funding}

Funding for this study was provided by the Agency for Healthcare Research and Quality (AHRQ), Healthcare Cost and Utilization Project (HCUP) (Contract No. HHSA-290-2013-00002-C). The views expressed in this article are those of the authors and do not necessarily reflect those of the Agency for Healthcare Research and Quality or the U.S. Department of Health and Human Services.

\section{Availability of data and materials}

The intramural datasets generated and/or analyzed during the current study are not publicly available. However, public-use State Inpatient Databases (SID), State Emergency Department Databases (SEDD), and State Ambulatory Surgery and Services Databases (SASD) are available through the Healthcare Cost and Utilization Project Central Distributor: https://www.hcup-us.ahrq. gov/databases.jsp.

\section{Ethics approval and consent to participate}

The HCUP databases used for this study are de-identified when received by the researchers and no one on the study team has any access to any identification information. AHRQ's Human Protections Administrator has determined this project does not constitute research involving human subjects. The HCUP data are not medical records, but hospital discharge billing records that are collected by hospitals, sent to statewide data agencies, and uniformly formatted by AHRQ. The data is permitted to be used by AHRQ under provisions spelled out in a Memorandum of Agreement with each HCUP Partner, and researchers must abide by the terms spelled out in the HCUP Data Use Agreements (https://hcup-us.ahrq.gov/team/StateDUA.pdf). No additional administrative permissions were required to access and use the HCUP data.

\section{Consent for publication}

Not applicable.

\section{Competing interests}

The authors of this manuscript declare that they have no personal or financial competing interests to disclose.

\footnotetext{
Author details

${ }^{1}$ University of Pittsburgh School of Nursing, 3500 Victoria St, 315B, Pittsburgh, PA 15213, USA. ${ }^{2}$ RAND Corporation, 4570 Fifth Ave \#600, Pittsburgh, PA 15213, USA. ${ }^{3}$ RAND Corporation, 1776 Main Street, Santa Monica, CA 90401 USA. ${ }^{4}$ Cedars-Sinai Medical Center, 8700 Beverly Boulevard, Becker 113, Los Angeles, CA 90048, USA. 'IBM Watson Health, 5425 Hollister Ave, Suite 140, Santa Barbara, CA 93111, USA. ${ }^{6}$ ML Barrett, Inc., 13943 Boquita Drive, Del Mar, CA 92014, USA. 7 Affiliation during this investigation: Agency for Healthcare Research and Quality, Rockville, Maryland, USA. ${ }^{8}$ Present address: West Virginia University, School of Public Health, 64 Medical Center Drive, PO Box 9190, Morgantown, W 26506-9190, USA. ${ }^{9}$ Agency for Healthcare Research and Quality, 5600 Fishers Lane, Rockville, MD 20857, USA.
} 
Received: 11 April 2019 Accepted: 8 April 2020

Published online: 08 June 2020

\section{References}

1. Feng Z, Wright B, Mor V. Sharp rise in Medicare enrollees being held in hospitals for observation raises concerns about causes and consequences. Health Aff (Millwood). 2012;31(6):1251-9.

2. Wright B, O'Shea AM, Ayyagari $P$, et al. Observation rates at veterans' hospitals more than doubled during 2005-13, similar to Medicare trends. Health Aff (Millwood). 2015;34(10):1730-7.

3. Noel-Miller C, Lind K. Is observation status substituting for hospital readmission? http://healthaffairs.org/blog/2015/10/28/is-observation-statussubstituting-for-hospital-readmission/. Accessed 20 Dec 2018.

4. Sheehy AM, Graf B, Gangireddy S, et al. Hospitalized but not admitted: characteristics of patients with "observation status" at an academic medical center. JAMA Internal Med. 2013;173(21):1991-8.

5. Amsterdam EA, Wenger NK, Brindis RG, et al. 2014 AHA/ACC guideline for the management of patients with non-ST-elevation acute coronary syndromes: a report of the American College of Cardiology/American Heart Association task force on practice guidelines. Circulation. 2014;130(25):e344-426.

6. Swap CJ, Nagurney JT. Value and limitations of chest pain history in the evaluation of patients with suspected acute coronary syndromes. JAMA 2005;294(20):2623-9.

7. Kontos MC, Diercks DB, Kirk JD. Emergency department and office-based evaluation of patients with chest pain. Mayo Clin Proc. 2010;85(3):284-99.

8. Taubenhaus $L$, Robilotti GD. The holding area: new arm of the ED. JACEP. 1972;1(4):15-9.

9. Bobzien WF. The observation-holding area: a prospective study. JACEP. 1979;8(12):508-12

10. Goodacre S, Cross E, Lewis C, et al. Effectiveness and safety of chest pain assessment to prevent emergency admissions: ESCAPE cluster randomised trial. BMJ. 2007;335(7621):659

11. Goodacre S, Nicholl J, Dixon S, et al. Randomised controlled trial and economic evaluation of a chest pain observation unit compared with routine care. BMJ. 2004;328(7434):254.

12. Roberts RR, Zalenski RJ, Mensah EK, et al. Costs of an emergency department-based accelerated diagnostic protocol vs hospitalization in patients with chest pain: a randomized controlled trial. JAMA. 1997;278(20): 1670-6.

13. Zalenski RJ, McCarren M, Roberts R, et al. An evaluation of a chest pain diagnostic protocol to exclude acute cardiac ischemia in the emergency department. Arch Intern Med. 1997;157(10):1085-91.

14. Baugh CW, Schuur JD. Observation care-high-value care or a cost-shifting loophole? N Engl J Med. 2013;369(4):302-5.

15. Barrett ML, Ringer KR, Owens PL, et al. Identifying Observation Services in the Healthcare Cost and Utilization Project (HCUP) State Databases. HCUP Methods Series Report \#2015-05. Agency for Healthcare Research and Quality. Published Sept 1, 2015. https://www.hcup-us.ahrq.gov/reports/ methods/2015-05_public.pdf.

16. Centers for Medicare and Medicaid Services. Medicare Benefit Policy Manual. Chapter 6, Hospital Services Covered Under Part B. https://www. cms.gov/Regulations-and-Guidance/Guidance/Manuals/downloads/bp102 c06.pdf. Accessed 20 Dec 2018

17. Zhao L, Schur C, Kowlessar N, et al. Rapid growth in Medicare hospital observation services: What's going on? AARP public policy institute report \#2013-10. Published Sept 2013. http://www.aarp.org/content/dam/aarp/ research/public_policy_institute/health/2013/rapid-growth-in-medicarehospital-observation-services-AARP-ppi-health.pdf.

18. Venkatesh AK, Geisler BP, Gibson Chambers JJ, et al. Use of observation care in US emergency departments, 2001 to 2008. PLoS One. 2011;6(9):e24326.

19. Venkatesh AK, Suter LG. Observation "services" and observation "care" - one word can mean a world of difference. Health Serv Res. 2014;49(4):1083-7.

20. Ross MA, Hemphill RR, Abramson J, Schwab K, Clark C. The recidivism characteristics of an emergency department observation unit. Ann Emerg Med. 2010;56(1):34-41.

21. Dharmarajan K, Qin L, Bierlein M, Choi JES, Lin Z, Desai NR, et al. Outcomes after observation stays among older adult Medicare beneficiaries in the USA: retrospective cohort study. BMJ. 2017;357:j2616.

22. Chin DL, Bang H, Manickam RN, Romano PS. Rethinking thirty-day hospital readmissions: shorter intervals might be better indicators of quality of care. Health Aff (Millwood). 2016;35(10):1867-75.
23. Graham KL, Wilker EH, Howell MD, et al. Differences between early and late readmissions among patients: a cohort study. Ann Intern Med. 2015;162(11): $741-9$.

24. Obermeyer Z, Cohn B, Wilson M, et al. Early death after discharge from emergency departments: analysis of national US insurance claims data. BMJ. 2017;356:j239.

25. Sabbatini AK, Kocher KE, Basu A, Hsia RY. In-hospital outcomes and costs among patients hospitalized during a return visit to the emergency department. JAMA. 2016;315(7):663-71.

26. Healthcare Cost and Utilization Project (HCUP). HCUP Home. Agency for Healthcare Research and Quality Web site. Updated November 2017. https://www.hcup-us.ahrq.gov/. Accessed 20 Dec 2018

27. Healthcare Cost and Utilization Project (HCUP). HCUP Databases. Agency for Healthcare Research and Quality Web site. Updated April 2017. https:// www.hcup-us.ahrq.gov/databases.jsp. Accessed 20 Dec 2018.

28. Barrett M, Steiner C, Andrews R, Kassed C, Nagamine M. Methodological Issues when Studying Readmissions and Revisits Using Hospital Adminstrative Data. 2011. HCUP methods series report \# 2011-01. ONLINE March 9, 2011. U.S. Agency for Healthcare Research and Quality. http:// www.hcupus.ahrq.gov/reports/methods/methods.jsp. Accessed 9 July 2019.

29. Wright B, O'Shea AM, Glasgow JM, et al. Outcomes associated with observation versus short-stay admission among chest pain patients in the veterans health administration. BMC Emerg Med. 2016;16(1):38.

30. Healthcare Cost and Utilization Project. Elixhauser comorbidity software. Agency for Healthcare Research and Quality Web site. Updated June 2017. www.hcup-us.ahrq.gov/toolssoftware/comorbidity/comorbidity.jsp. Accessed 20 Dec 2018.

31. lezzoni L. Risk adjustment for measuring health care outcomes. 4th ed. Chicago, IL: Health Administration Press; 2013.

32. Reinhardt SW, Lin CJ, Novak E, et al. Noninvasive cardiac testing versus clinical evaluation alone in acute chest pain: a secondary analysis of the ROMICAT-II randomized clinical trial. JAMA Intern Med. 2018;178(2):212 published online November 14, 2017.

33. Backus BE, Six AJ, Kelder JH, et al. Risk scores for patients with chest pain: evaluation in the emergency department. Curr Cardiol Rev. 2011;7(1):2-8.

34. Fruergaard $P$, Launbjerg J, Hesse B, et al. The diagnoses of patients admitted with acute chest pain but without myocardial infarction. Eur Heart J. 1996;17(7):1028-34.

35. Ruddox V, Mathisen M, Otterstad JE. Prevalence and prognosis of nonspecific chest pain among patients hospitalized for suspected acute coronary syndrome - a systematic literature search. BMC Med. 2012;10:58.

36. Cafardi SG, Pines JM, Deb $P$, et al. Increased observation services in Medicare beneficiaries with chest pain. Am J Emerg Med. 2016;34(1):16-9.

\section{Publisher's Note}

Springer Nature remains neutral with regard to jurisdictional claims in published maps and institutional affiliations.

Ready to submit your research? Choose BMC and benefit from:

- fast, convenient online submission

- thorough peer review by experienced researchers in your field

- rapid publication on acceptance

- support for research data, including large and complex data types

- gold Open Access which fosters wider collaboration and increased citations

- maximum visibility for your research: over $100 \mathrm{M}$ website views per year

At $\mathrm{BMC}$, research is always in progress.

Learn more biomedcentral.com/submissions 